\title{
Bevacizumab and Breast Cancer: A Meta-Analysis of First-Line Phase III Studies and a Critical Reappraisal of Available Evidence
}

\author{
José R. Rossari, ${ }^{1}$ Otto Metzger-Filho, ${ }^{1}$ Marianne Paesmans, ${ }^{1}$ Kamal S. Saini, ${ }^{1}$ \\ Alessandra Gennari, ${ }^{2}$ Evandro de Azambuja, ${ }^{1}$ and Martine Piccart-Gebhart ${ }^{1}$ \\ ${ }^{1}$ Institut Jules Bordet, Université Libre de Bruxelles, Boulevard de Waterloo, 125, 1000 Brussels, Belgium \\ ${ }^{2}$ Medical Oncology, Galliera Hospital, Via Volta 6, 16125 Genoa, Italy \\ Correspondence should be addressed to Martine Piccart-Gebhart, martine.picart@bordet.be
}

Received 4 May 2012; Accepted 1 August 2012

Academic Editor: Maryam Lustberg

Copyright (C) 2012 José R. Rossari et al. This is an open access article distributed under the Creative Commons Attribution License, which permits unrestricted use, distribution, and reproduction in any medium, provided the original work is properly cited.

Background. Randomized studies have shown different magnitude of bevacizumab benefit in the treatment of advanced breast cancer. Regulatory agencies have modified bevacizumab treatment indications across different regions. In this study, we perform a meta-analysis of phase III studies aiming to interrogate the magnitude of bevacizumab benefit for the treatment of first-line HER2negative metastatic breast cancer (MBC). Methods. Data from studies E2100, AVADO and RIBBON-1 were used to calculate the benefit of bevacizumab in terms of tumor overall response rate (ORR), progression-free survival (PFS), overall survival (OS), and toxicities. Combined statistical estimates of hazard ratios (HR) and odds ratios were calculated using fixed-effects or randomeffects models. Results. A total of 2,695 patients were evaluated. Combining bevacizumab with different chemotherapy backbones resulted in a $30 \%$ risk reduction of PFS events (HR $=0.70 ; 95 \%$ confidence interval $[\mathrm{CI}], 0.57-0.86)$ and increased ORR (odds ratio $1.81 ; 95 \% \mathrm{CI}, 1.53-2.14)$. No OS benefit could be demonstrated (HR $=0.95 ; 95 \% \mathrm{CI}, 0.85-1.06)$. Bevacizumab significantly increased the incidence of adverse events such as proteinuria, hypertension and cardiovascular events. Conclusions. Bevacizumab combined with chemotherapy in the first-line treatment of MBC significantly improved ORR and PFS, but also increased grade 3-4 toxicities. No significant OS advantage was observed.

\section{Introduction}

Vascular endothelial growth factor (VEGF) and its receptors are thought to play a pivotal role in tumor angiogenesis [1]. Bevacizumab is a humanized monoclonal antibody designed to block VEGF-A and has proved to be effective in colorectal cancer, nonsmall cell lung cancer, renal cell carcinoma, ovarian carcinoma, and glioblastoma multiforme [2-8].

In the field of breast cancer, bevacizumab has generated more controversies and discussions than any other targeted therapy. In February 2008, the FDA granted accelerated approval to bevacizumab in combination with paclitaxel for the first-line treatment of metastatic, HER2-negative metastatic breast cancer (MBC), based on promising results of the Eastern Cooperative Oncology Group (ECOG) 2100 trial. On July 20th 2010, the Oncologic Drugs Advisory
Committee (ODAC) of the FDA's Center for Drug Evaluation and Research voted 12 to 1 against the use of bevacizumab in combination with chemotherapy for the first-line treatment of advanced breast cancer [9]. This was followed by a definitive announcement by the FDA revoking approval of bevacizumab for this indication [10].

Nonetheless bevacizumab is still approved by different regulatory agencies across several countries as a standard antiangiogenic drug for the treatment of first-line advanced breast cancer. With more than 20,000 breast cancer patients currently being randomized into bevacizumab studies, it is crucial to define which magnitude of endpoint or risk/benefit ratio is expected. In this study, we performed a meta-analysis of randomized phase III studies evaluating bevacizumab for the first-line treatment of metastatic breast cancer [11-13]. The magnitude of risks and benefits of adding bevacizumab 
to the standard treatment of advanced cancer are discussed in the context of recent controversies and ongoing randomized phase III clinical trials.

\section{Methods}

2.1. Study Selection and Data Extraction. MEDLINE searches were performed to identify eligible studies, which were restricted to phase III, randomized, controlled trials comparing the combination of bevacizumab to chemotherapy with chemotherapy alone for the first-line treatment of mostly HER2-negative advanced breast cancer. The proceedings of the San Antonio Breast Cancer Symposium, European Society of Medical Oncology, and American Society of Clinical Oncology annual meetings were examined for presented abstracts. Based on these criteria, the E2100, AVADO, and RIBBON-1 were selected for our meta-analysis.

2.2. Data Extraction. Data abstraction was conducted independently by three investigators (J. R. Rossari, O. MetzgerFilho, and M. Paesmans) in accordance with the Preferred Reporting Items for Systematic Reviews and Meta-Analyses (PRISMA) guidance [14]. For each study the following information was extracted: publication or presentation date, first author's last name, sample size, primary endpoints, regimens used, dosage and scheduling of chemotherapy and bevacizumab, line of treatment, number of chemotherapy cycles, additional treatments given (regardless of study arm), follow-up period, number of outcome events, information pertaining to study design, PFS definition, tumor response criteria, data on PFS, ORR and OS, subgroup evaluation, quality of life analysis, crossover, if any, and toxicities.

2.3. Statistical Methods. The impact on PFS and OS of adding bevacizumab to a chemotherapy regimen was measured in terms of the hazard ratio (HR). For each study, the HR was either extracted directly from the reports, or it was estimated. Two of the studies included in this meta-analysis were designed to compare more than one bevacizumab arm with a control group, either to evaluate different doses of the drug (AVADO), or to evaluate its combination with different cytotoxic agents (RIBBON-1). To render the statistical analysis of these comparisons feasible, each analysis was treated as a different study, and a HR was extracted from all of them. Whenever possible, HR was also estimated for PFS according to subgroups of patients defined a priori (age, hormone receptor status, prior adjuvant chemotherapy, prior taxane therapy, and length of disease-free interval [DFI]). For some subgroups, it was necessary to extrapolate HRs and their variances from graphical representations [15]. Individual $\mathrm{HR}$ estimates were then combined into overall $\mathrm{HR}$ using fixed effects or random effects models, depending on the outcome of the heterogeneity $X^{2}$-test. If heterogeneity was not detected (at the 10\% significance level), the fixedeffects model was applied. Heterogeneity was quantified by the $I^{2}$ coefficient that measures the percentage of total variation across studies that is due to heterogeneity rather than chance [16]. By convention, a HR $<1.00$ implied a benefit of adding bevacizumab to a chemotherapy regimen. This impact was considered statistically significant if the $95 \%$ confidence interval (CI) for overall HR did not overlap 1.00 [17].

The association of bevacizumab with toxicities and response rate were calculated in terms of odds ratios, applying the same statistical methods described above.

\section{Results}

3.1. First-Line Bevacizumab Studies in Metastatic Breast Cancer. Three randomized phase III studies evaluating the impact of adding bevacizumab to chemotherapy in the first-line treatment of HER2-negative MBC have reported positive results in terms of overall response rate (ORR) and progression-free survival (PFS). E2100, AVADO, and RIBBON-1 are the focus of this meta-analysis and the key results of the three trials are summarized in Table 1 [11-13]. The E2100 study showed that the addition of biweekly bevacizumab $10 \mathrm{mg} / \mathrm{kg}$ to weekly paclitaxel doubled the PFS when compared with paclitaxel alone in the first-line treatment of patients with HER2-negative MBC without an overall survival (OS) gain [11]. Treatment was continued until unacceptable toxicity or disease progression and no crossover was allowed. Subsequently, the AVADO study randomized patients to docetaxel alone or in combination to bevacizumab (at two dose levels of $15 \mathrm{mg} / \mathrm{kg}$ or $7.5 \mathrm{mg} / \mathrm{kg}$ ) and demonstrated improvement in PFS and no OS benefit. In the AVADO study treatment was continued for up to nine weeks, disease progression, or unacceptable toxicity. In contrast to E2100, $40 \%$ of patients enrolled on AVADO received bevacizumab in the second-line setting. The RIBBON-1 trial in which bevacizumab $15 \mathrm{mg} / \mathrm{kg}$ was added to capecitabine, anthracycline, or taxane-based chemotherapy similarly showed improved PFS without OS benefit [13]. Approximately $60 \%$ of patients randomized in the RIBBON-1 trial received bevacizumab as second-line chemotherapy. The PFS gain observed in the E2100 trial was not replicated with the same magnitude in the subsequent phase III studies (AVADO and RIBBON-1), and OS could not be demonstrated.

The three studies selected for this meta-analysis represented a total of 2,695 patients, with the number of patients in each study ranging from 722 to 1,237 . The AVADO and RIBBON-1 trials were double-blinded, placebo-controlled studies, while the E2100 trial was an open-labeled study with patients randomly assigned to paclitaxel alone or the combination of paclitaxel and bevacizumab. Median followup times were provided only in AVADO and RIBBON-1 studies.

\subsection{Combined Analysis of First Line Studies (E2100, AVADO, and Ribbon-1)}

3.2.1. Progression-Free Survival. Figure 1 shows the HR for PFS in each individual trial and the overall analysis. The HR for PFS of the bevacizumab arms were evaluated separately and compared to the control arms in the AVADO and 
TABle 1: Phase III studies with bevacizumab and chemotherapy as first-line treatment of metastatic breast cancer.

\begin{tabular}{|c|c|c|c|c|c|c|c|}
\hline Study & Treatment line & Arms & Patients & Response rate & $\begin{array}{l}\text { Progression-free } \\
\text { survival }\end{array}$ & Overall survival & Crossover \\
\hline $\begin{array}{l}\text { E2100 } \\
(2007)\end{array}$ & First & $\begin{array}{l}\text { Paclitaxel q1w +/- } \\
\text { Bev } 10 \mathrm{mg} / \mathrm{kg} \mathrm{q2w}\end{array}$ & 722 & $\begin{array}{c}\text { 36.9\% versus } \\
21.2 \% \\
(P<0.001)\end{array}$ & $\begin{array}{l}\mathbf{1 1 . 8} \text { versus } 5.9 \\
\text { months [HR } 0.6 \\
(0.51-0.7)]\end{array}$ & $\begin{array}{l}26.7 \text { versus } 25.2 \\
\text { months [HR } 0.88 \\
(P=0.16)]\end{array}$ & Not allowed \\
\hline $\begin{array}{l}\text { AVADO } \\
(2008)\end{array}$ & First & $\begin{array}{l}\text { Docetaxel } \mathrm{q} 3 \mathrm{w}+\mathrm{Bev} \\
15 \mathrm{mg} / \mathrm{kg} \text { or Bev } \\
7.5 \mathrm{mg} / \mathrm{kg} \text { or Placebo } \\
\mathrm{q} 3 \mathrm{w}\end{array}$ & 736 & $\begin{array}{c}\mathbf{6 4} \%(P<0.001) \\
\text { versus } \mathbf{5 5} \% \\
(P=0.07) \\
\text { versus } \mathbf{4 6} \%\end{array}$ & $\begin{array}{l}\mathbf{1 0 . 1}[\text { HR } 0.77 \\
(0.64-0.93)] \text { versus } \\
\mathbf{9 . 0}[\text { HR } 0.86 \\
(0.72-1.04)] \text { versus } \\
\mathbf{8 . 2} \text { months }\end{array}$ & $\begin{array}{l}\mathbf{3 0 . 2}[\text { HR } 1.03 \\
(0.7-1.3)] \text { versus } \\
\mathbf{3 0 . 8}[\text { HR } 1.05 \\
(0.81-1.36)] \text { versus } \\
\mathbf{3 1 . 9} \text { months }\end{array}$ & Allowed \\
\hline $\begin{array}{l}\text { RIBBON } 1 \\
(2009)\end{array}$ & First & $\begin{array}{l}\text { Capecitabine q3w + } \\
\text { Bev } 15 \mathrm{mg} / \mathrm{kg} \mathrm{q} 3 \mathrm{w} \text { or } \\
\text { Placebo q3w } \\
\text { Anthracycline } / \\
\text { Taxane }^{2} \mathrm{q} 3 \mathrm{w}+\mathrm{Bev} \\
15 \mathrm{mg} / \mathrm{kg} \mathrm{q} 3 \mathrm{w} \text { or } \\
\text { Placebo q3w }\end{array}$ & 1,237 & $\begin{array}{c}\text { 35.4\% versus } \\
\mathbf{2 3 . 6} \% \\
(P=0.009) \\
\mathbf{5 1 . 3} \% \text { versus } \\
\mathbf{3 7 . 9} \% \\
(P=0.005)\end{array}$ & $\begin{array}{l}\mathbf{8 . 6} \text { versus } \mathbf{5 . 7} \\
\text { months }[\text { HR } 0.69 \\
(0.56-0.84)] \\
\mathbf{9 . 2} \text { versus } \mathbf{8 . 0} \\
\text { months [HR } 0.64 \\
(0.52-0.80)]\end{array}$ & $\begin{array}{l}\mathbf{2 9 . 0} \text { versus } 21.2 \\
\text { months [HR } 0.85 \\
(0.63-1.14)] \\
\mathbf{2 5 . 2} \text { versus } 23.8 \\
\text { months [HR } 1.03 \\
(0.77-1.38)]\end{array}$ & Allowed \\
\hline
\end{tabular}

${ }^{1}$ Adriamycin or Epirubicin + Cyclophosphamide +/- 5-Fluorouracil q3w; ${ }^{2}$ Docetaxel or nab-Paclitaxel q3w.

Abbreviations: ORR: overall response rate; PFS: progression-free survival; HR: hazard ratio; LD: low dose; HD: high dose.

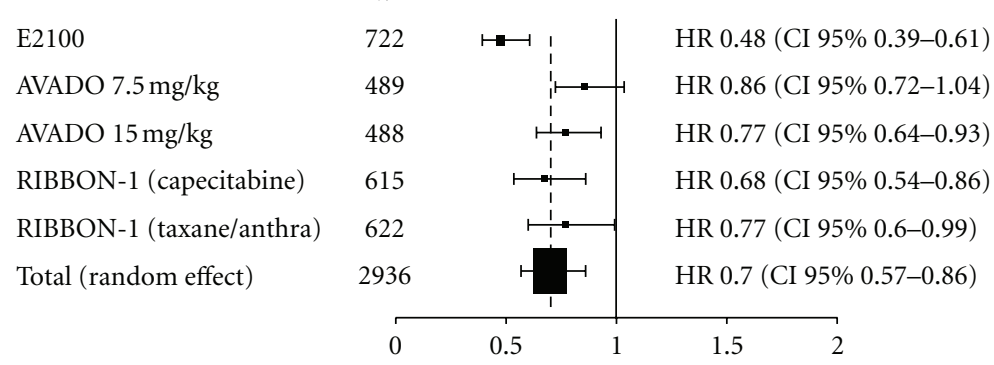

FIgURE 1: Progression-free survival hazard ratios. Abbreviations: Anthra: anthracycline; $n$ : number of patients.

RIBBON-1 trials. Individually, a consistent PFS benefit was observed across all three trials. Our meta-analysis shows a statistically significant benefit obtained by adding bevacizumab to chemotherapy in the first-line treatment of MBC patients: the overall HR was 0.70 (95\% CI, 0.57 to 0.86 ), corresponding to a $30 \%$ reduction of the hazard of progression for bevacizumab-based regimens. Statistically significant heterogeneity was observed between the studies $(P=0.0006), I^{2}=77 \%$.

3.2.2. Progression-Free Survival according to Subgroups. PFS was assessed according to hormone receptor status (positive or negative), prior adjuvant chemotherapy (yes or no), age ( $<65$ years versus $\geq 65$ years), use of prior taxane (yes or no), and DFI (short or long), although the definition of DFI differed between the trials. For instance, AVADO and E2100 trials stratified DFI in $\leq 24$ months versus $>24$ months, while RIBBON-1 considered $\leq 12$ months versus $>12$ months.

The addition of bevacizumab to chemotherapy consistently showed a PFS benefit in all analyzed subgroups, as shown in Figure 2. Interaction tests were carried out and did not reveal any significant interaction between analyzed covariates and bevacizumab effect $(P=0.74)$.
3.2.3. Overall Survival. Figure 3 shows the HR for OS in each individual trial and the overall analysis. As was the case with PFS, the HRs of the clinical trials with two bevacizumab arms (AVADO and RIBBON-1) were evaluated separately, and each was compared to the control group. Individually, none of the studies showed a significant OS benefit of adding bevacizumab to chemotherapy as first-line treatment of MBC. Our results show no statistically significant benefit of adding bevacizumab to chemotherapy in the first-line treatment of MBC patients (HR 0.95, 95\% CI, 0.85 to 1.06). No heterogeneity was identified between the trials $(P=$ $0.65), I^{2}=0 \%$.

3.2.4. Overall Response Rate. As shown in Figure 4, the odds ratio of response associated with the addition of bevacizumab to chemotherapy was 1.81 (95\% CI, 1.53-2.14). Again, no heterogeneity was seen between trials $(P=0.55)$, $I^{2}=0 \%$. The results did not change when the arm of the RIBBON-1 trial containing capecitabine (and no taxane) was removed from the analysis (odds ratio 1.83 ; 95\% CI, $1.52-$ 2.19; $P$ test for heterogeneity 0.39 ) to evaluate the effect of adding bevacizumab to a taxane-based chemotherapy. 


\begin{tabular}{lcll|l|} 
Subgroups & Pts & HR $(95 \% \mathrm{CI})$ \\
ER positive & 1156 & $0.77(0.7-0.84)$ \\
ER and PgR negative & 449 & $0.67(0.57-0.78)$ \\
$<65$ years & 1337 & $0.67(0.57-0.78)$ & \\
$\geq 65$ years & 313 & $0.75(0.62-0.91)$ & \\
Short DFI & 1019 & $0.65(0.55-0.76)$ & \\
Long DFI & 1868 & $0.7(0.63-0.78)$ & \\
No prior adjuvant chemo & 1083 & $0.76(0.66-0.88)$ & \\
Prior adjuvant chemo & 1804 & $0.65(0.58-0.73)$ & \\
No prior taxane & 1392 & $0.75(0.67-0.85)$ & & \\
Prior taxane & 749 & $0.51(0.39-0.66)$ & 0.5 & 1
\end{tabular}

FIgURE 2: Progression-free survival hazard ratios across subgroups. Abbreviations: chemo: chemotherapy, DFI: disease-free interval, ER: estrogen receptor, PgR: progesterone receptor.

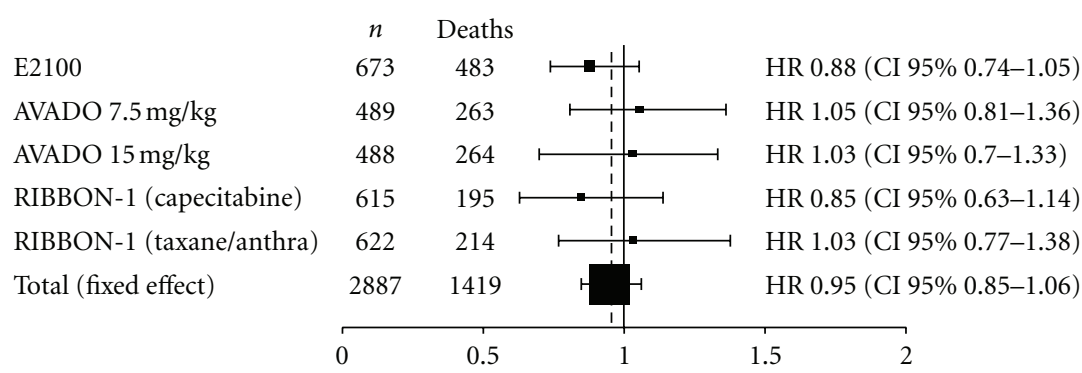

FIGURE 3: Overall survival hazard ratios. Abbreviations: Anthra: anthracycline, $n$ : number of patients.

3.2.5. Safety Profile of Bevacizumab. The addition of bevacizumab to chemotherapy increased the probability of grade 3-4 hypertension (random effects odds ratio 5.56; 95\% CI, 1.66-18.62), proteinuria (fixed effects odds ratio 5.35; 95\% CI, 2.80-10.20), sensory neuropathy (fixed effects odds ratio $1.48 ; 95 \% \mathrm{CI}, 1.11-1.99)$, and cardiac events including left ventricular (LV) dysfunction and congestive heart failure (fixed effects odds ratio 3.36; 95\% CI, $1.41-$ 8.01). No significant increase in the risk of gastrointestinal (GI) perforation was seen in MBC patients treated with bevacizumab (fixed effects odds ratio 0.94 ; 95\% CI, $0.31-$ 2.85) [18].

\section{Discussion}

Bevacizumab combined with chemotherapy in the first-line treatment of MBC significantly improved ORR and PFS, but also increased grade 3-4 toxicities. No significant OS advantage was observed. One of the most frequently cited reasons for conducting a meta-analysis is the increase in statistical power that it affords; however, inherent limitations may limit the accuracy of results. In this study, we acknowledge the following limitations: First, it was conducted using published study results, rather than individual patient data. Second, for the two studies with more than one bevacizumab containingarm, control arms had to be duplicated in order to give each comparison independent statistical treatment. Third, despite being the primary endpoint in all three trials, the definition of PFS was not precisely specified in all of them. Despite limitations our results provided similar conclusions when compared to other studies [19-22]. Three meta-analyses evaluated the efficacy of bevacizumab plus chemotherapy for the treatment of $\mathrm{MBC}$, and provide interesting points of comparison with our study [19-21]. Valachis et al. analyzed five studies, including a phase II study and one trial with bevacizumab and capecitabine after first-line chemotherapy for MBC, and found global HRs similar to the ones we report: 0.70 (95\% CI $0.60-0.82)$ for PFS and $0.90(95 \%$ CI 0.80-1.03) for OS [19]. Lee et al. analyzed four studies involving a total of 2,860 patients, to verify the clinical efficacy of bevacizumab in the salvage treatment of MBC, and reported PFS (HR 0.69, 95\% CI, 0.58-0.81), OS (HR 0.92, 95\% CI, 0.82-1.03), and ORR (HR 1.53, 95\% CI, 1.371.71) [20]. O' Shaughnessy et al. conducted a meta-analysis including individual patient data from the E2100, AVADO, and RIBBON-1 studies and showed a $36 \%$ reduction in the risk of a PFS event $(\mathrm{HR}=0.64,95 \%$ CI $0.57-0.71)$ and no median OS gain ( $\mathrm{HR}=0.97 ; 95 \%$ CI 0.86-13.08) [21]. However, one-year survival rate was statistically significant increased for patients treated in the bevacizumab-arms $(77 \%$ versus $82 \%, P=0.003$ ) [21].

In this study, the addition of bevacizumab to chemotherapy statistically increased PFS in all analyzed subgroups. Importantly, HER2-positive disease was not allowed in the AVADO and RIBBON-1 studies, and only about $1 \%$ of 


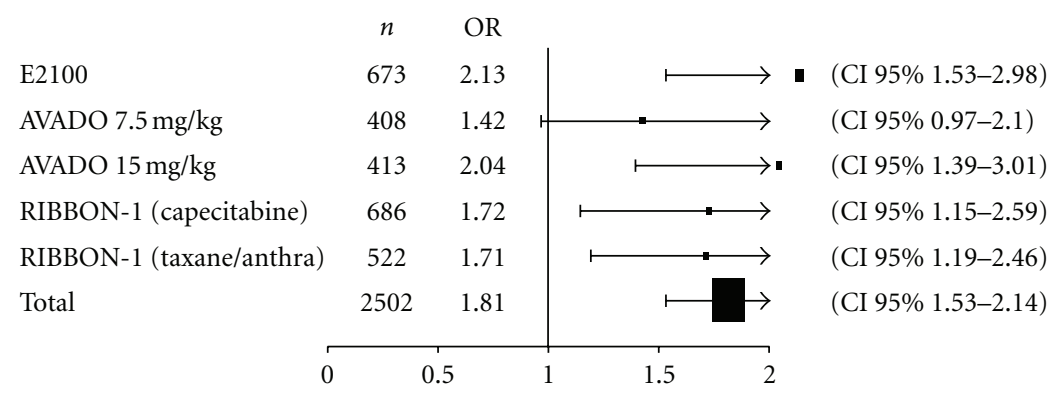

FIGURE 4: Overall response rate (ORR) Odds Ratio (OR). Abbreviations: Anthra, anthracycline; $n$, number of patients.

patients participating in E2100 had HER2 overexpressing tumors. Our results seem to concur with those obtained by O'Shaughnessy et al. in a comparison of the subgroup analyses performed in the same studies [23]. Similar PFS benefit with the addition of bevacizumab was observed in patients according to hormone receptor-negative status, age, and previous exposure to taxane. Comparing with their meta-analysis, beyond efficacy in terms of PFS and OS, our study also adds relevant information about the safety profile of bevacizumab in this population of patients.

The results of individual bevacizumab phase III studies and meta-analyses motivated important discussions about adequate endpoints for breast cancer studies. Whether PFS benefit can be used as a surrogate for OS in breast cancer patients is a matter of debate, and OS continues to be the endpoint of choice to assess the efficacy of new treatments for patients with MBC [24]. The availability of bevacizumab as second-line treatment for some patients enrolled in the AVADO and RIBBON-1 studies might therefore be considered a confounding factor, which could in turn have affected the chance of demonstrating an OS benefit in the intent-to-treat analysis.

An earlier phase III study failed to demonstrate PFS benefit when bevacizumab was added to capecitabine for patients with $\mathrm{MBC}$ previously treated with both an anthracycline and a taxane, and at least one prior chemotherapy regimen for metastatic disease [25]. That finding has been recently challenged by the results of the RIBBON-2 trial, which demonstrated ORR and PFS gains with the addition of bevacizumab to second-line chemotherapy [26]. If the benefits of bevacizumab are extended to subsequent lines of treatment after first disease progression, crossover is likely to pose an important obstacle for detecting OS gains in first-line chemotherapy.

Recently, the results of two large neoadjuvant clinical trials evaluating the addition of bevacizumab to different chemotherapy regimens further increased the controversies surrounding bevacizumab therapy for breast cancer [10]. Both the National Surgical Adjuvant Breast and Bowel Project (NSABP) B-40 [27] and the GeparQuinto (GBG44) [28] were designed to evaluate whether the addition of bevacizumab to chemotherapy would increase the rates of Pathologic Complete Response (PCR) in women with earlystage HER2-negative breast cancer. In both studies, the rates of PCR defined as the absence of invasive disease in the breast, irrespective of nodes, were in favor of bevacizumab (16.5\% without bevacizumab versus $20.5 \%$ with bevacizumab in the GBG44 trial, and $28.2 \%$ versus $34.5 \%$ in the NSABP B-40 trial). However, neither trial showed significant differences when PCR was defined as the absence of invasive disease in the breast and lymph nodes (GBG44: $18.3 \%$ versus $21.7 \%, P=0.07$; NSABP B-40: $23.0 \%$ vresus $27.6 \%, P=0.08$, both resp. without and with bevacizumab). In contrast to our results and previous findings [23], subgroup analysis of the NSABP B40 trial and GBG44 studies suggested differential benefit of bevacizumab according to breast cancer subtypes [27, 28]. Intriguingly, the results were not in the same direction. In the GBG44, the rates of PCR were increased with bevacizumab in patients with hormone receptor-negative, while the NSABP B-40 showed increased PCR in the with hormone receptorpositive cancers.

In the current analysis, bevacizumab therapy was associated with increased proteinuria, hypertension, cardiovascular dysfunction, and sensory neuropathy. In agreement with our findings, a combined analysis of five phase III trials in advanced breast cancer showed a statistically significant increase in proteinuria $(\mathrm{OR}=27.68)$, hypertension $(\mathrm{OR}=12.76)$, and left ventricular dysfunction (LVD) $(\mathrm{OR}=2.25)$ with the addition of bevacizumab [29]. In addition, hemorrhagic events $(\mathrm{OR}=4.07)$ were also associated to bevacizumab [29]. Moreover, several metaanalyses have been reported linking specific adverse events to bevacizumab therapy. Gastrointestinal perforation was associated to bevacizumab therapy among 12,294 patients evaluated with a relative risk (RR) of 2.14 (95\% CI 1.193.85 ), but statistically significant only for colorectal cancer patients [18]. Hypertension was associated to bevacizumab therapy in a meta-analysis conducted among 12,049 patients across several tumor subtypes with a RR of 5.38 (95\% CI 3.63-7.97) [30]. Venous thromboembolism was associated to bevacizumab therapy among 7,956 patients studied with a RR of 1.33 (95\% CI 1.13-1.56), but not significant in the breast cancer subset [31]. Arterial thromboembolic events including cardiac ischemia was increased in a meta-analysis including 12,617 patients with a RR of 1.44 (95\% CI $1.08-$ 1.91). An additional study found an incidence of arterial thromboembolism of $0.7 \%$ in BC patients treated with bevacizumab, which was not significantly higher when compared to chemotherapy alone ( $\mathrm{RR}=1.47,95 \% \mathrm{CI} 09.27-7.95)$ [32]. 
TABLE 2: Ongoing a phase III clinical trials evaluating the addition of bevacizumab for the treatment of advanced breast cancer.

\begin{tabular}{|c|c|c|c|}
\hline Identifier & Setting & $n$ & Study Design \\
\hline NCT00600340 & Advanced & 560 & Bevacizumab + paclitaxel versus bevacizumab + capecitabine \\
\hline NCT01303679 & Advanced & 198 & Bevacizumab + exemestane versus bevacizumab + paclitaxel \\
\hline NCT01131195 & Advanced & 142 & Bevacizumab + paclitaxel versus metronomic ctx + capecitabine \\
\hline NCT01250379 & Advanced & 488 & Chemotherapy + bevacizumab. versus chemotherapy* \\
\hline NCT00929240 & Advanced & & Bevacizumab + capecitabine. versus bevacizumab ${ }^{* *}$ \\
\hline NCT00785291 & Advanced & 900 & $\begin{array}{l}\text { Bevacizumab + paclitaxel versus paclitaxel } \\
\text { Bevacizumab + nab-paclitaxel versus nab-paclitaxel } \\
\text { Bevacizumab + ixabepilone versus ixabepilone }\end{array}$ \\
\hline NCT01200212 & Advanced & 432 & Bevacizumab + taxane + capecitabine versus taxane + capecitabine \\
\hline NCT00545077 & Advanced & 378 & Letrozole or fulvestrant versus letrozole or fulvestrant + bevacizumab \\
\hline NCT00601900 & Advanced & 502 & Tamoxifen or letrozole versus tamoxifen or letrozole + bevacizumab \\
\hline NCT00391092 & Advanced & 407 & Bevacizumab + trastuzumab + docetaxel versus trastuzumab + docetaxel \\
\hline NCT00520975 & Advanced & 489 & $\begin{array}{l}\text { Bevacizumab }+ \text { trastuzumab }+ \text { carboplatin }+ \text { paclitaxel versus trastuzumab }+ \text { carboplatin }+ \\
\text { paclitaxel }\end{array}$ \\
\hline
\end{tabular}

Identifiers are from clinicaltrials.gov website. ${ }^{*}$ Patients previously treated with bevacizumab. ${ }^{*}$ Patients treated with bevacizumab and docetaxel and no evidence of progressive disease.

Abbreviations: $n$ : estimated number of patients.

TABLE 3: Ongoing adjuvant phase III trials evaluating the addition of bevacizumab for the treatment of early breast cancer.

\begin{tabular}{|c|c|c|c|c|}
\hline Identifier & Study & $N$ & Study population & Study design \\
\hline NCT00528567 & BEATRICE & 2,583 & Triple negative BC & $\begin{array}{c}\text { Standard CT versus } \\
\text { Standard CT + BEV for } 1 \text { year }\end{array}$ \\
\hline NCT00887536 & NSABP B-46 & 3,900 & $\begin{array}{c}\text { HER2 negative } \\
\mathrm{N}+\text { or high risk } \mathrm{N}-\end{array}$ & $\begin{array}{c}\text { Docetaxel/cyclophosphamide } \times 6 \text { versus } \\
\text { Docetaxel/doxorubicin/cyclophosphamide } \times 6 \\
\text { versus } \\
\text { Docetaxel/cyclophosphamide/BEV } \times 6 \\
\text { (followed by BEV alone until } 1 \text { year) }\end{array}$ \\
\hline NCT00625898 & BETH & 3,509 & $\begin{array}{c}\text { HER2 positive } \\
\mathrm{N}+\text { or high risk } \mathrm{N}-\end{array}$ & $\begin{array}{c}\text { Docetaxel/carboplatin/TRAST } \times 6 \\
\text { (followed by TRAST alone until } 1 \text { year) versus } \\
\text { Docetaxel/carboplatin/TRAST/BEV } \times 6 \\
\text { (followed by TRAST/BEV until } 1 \text { year) versus } \\
\text { Docetaxel }[\times 3] / \text { FEC }[\times 3] / \text { TRAST } \times 6 \\
\text { (followed by TRAST alone until } 1 \text { year) versus } \\
\text { Docetaxel }[\times 3] / \text { FEC }[\times 3] / \text { TRAST/BEV } \times 6 \\
\text { (followed by TRAST/BEV until } 1 \text { year) }\end{array}$ \\
\hline NCT00433511 & ECOG 5103 & 4,950 & $\begin{array}{c}\text { HER2 negative } \\
\mathrm{N}+\text { or high risk } \mathrm{N}-\end{array}$ & $\begin{array}{c}\text { AC }[\times 4] / \text { weekly paclitaxel }[\times 12] / \text { placebo versus } \\
\text { AC }[\times 4] / \text { weekly paclitaxel }[\times 12] / \text { BEV versus } \\
\text { AC }[\times 4] / \text { weekly paclitaxel }[\times 12] / \text { BEV } \\
\text { (followed by BEV alone until } 1 \text { year) }\end{array}$ \\
\hline
\end{tabular}

Identifiers are from clinicaltrials.gov website.

Abbreviations: $n$ : estimated number of patients; $\mathrm{N}+$ : lymph nodes positive; $\mathrm{N}-:$ lymph nodes negative; CT: chemotherapy; BEV: bevacizumab; TRAST: trastuzumab; FEC: 5-fluorouracil/epirubicin/cyclophosphamide; AC: adriamycin/cyclophosphamide.

Serious congestive heart failure (CHF) was associated to bevacizumab therapy among 3,784 BC patients evaluated with a RR of 4.74 (95\% CI 1.66-11.18) [33]. A higher risk of fatal adverse event was associated with bevacizumab therapy among 10,217 patients evaluated $(\mathrm{RR}=1.46$, 95\% CI, $1.09-$ 1.94). However, in the subset of breast cancer studies no significant increase in fatal adverse events was observed $(\mathrm{RR}=0.69,95 \% \mathrm{CI}, 0.3-1.62)$ [34]. Fatal adverse events were attributed mainly to gastrointestinal perforation, neutropenia, and hemorrhage and more likely to occur among patients with pancreatic and lung cancer. For instance, in the Athena trial, which prospectively evaluated the safety of bevacizumab in combination to taxane regimens among $2,551 \mathrm{BC}$ patients [35], fatal adverse events occurred in $0.7 \%$ of patients.

Hence, a clear understanding of the magnitude of bevacizumab benefit, toxicity, and benefit across breast cancer subgroups is of paramount importance. Bevacizumab is under evaluation across several phase III studies and correct estimates of treatment related toxicities 
and efficacy from previous studies are fundamental to the appropriate guidance and conduct of ongoing studies (Table 2). In the advanced setting, phase III studies are estimated to enroll over 4,000 patients. Bevacizumab is being studied in combination to different chemotherapy regimens (NCT00600340, NCT01303679, NCT01131195, NCT00785291); as maintenance therapy (NCT01250379, NCT00929240); in combination with more than one cytotoxic drug (NCT01200212); in combination with trastuzumab (NCT00520975, NCT00391092); and in combination with hormonal therapy. Cancer and Leukemia Group B (CALGB) 40503 trial (NCT00601900) randomizes patient with locally advanced or metastatic BC to receive tamoxifen or letrozole, with or without bevacizumab. Similarly, another study (NCT00545077) randomizes postmenopausal patients with advanced BC for endocrine therapy (letrozole or fulvestrant) alone or in combination with bevacizumab. Importantly, a broad spectrum of phase III studies are expected to randomize about 15,000 early breast cancer patients into four large adjuvant studies (Table 3 ).

The recent decisions by FDA regarding use of bevacizumab in patients with MBC has turned the spotlight on the risk-versus-benefit of adding bevacizumab to chemotherapy [36]. Today, thousands of women with breast cancer are being randomized into bevacizumab studies; therefore, it is imperative to define which magnitude of endpoint or risk/benefit ratio is expected. Moreover, there is a great need to identify and validate biomarkers to aid clinical decisions in the treatment with antiangiogenic therapies. The side effects associated with bevacizumab are considerable and predictive biomarkers to identify subgroups most likely to benefit are needed. In a retrospective analysis conducted in the E2100 study, VEFG polymorphisms were able to predict not only bevacizumab benefit but also toxicity [37]. However, validation of biomarker findings in subsequent studies is needed and the incorporation of translational research questions into prospective clinical trials should be mandatory.

\section{Disclosure}

M. Piccart-Gebhart and E. de Azambuja are consultants and receive honoraria from Roche.

\section{Acknowledgment}

The authors would like to thank Carolyn Straehle for her editorial assistance. Presented in part at the European Breast Cancer Conference (EBCC)-7, April 24-27, 2010, Barcelona, Spain (abstract no. 122).

\section{References}

[1] S. Banerjee, M. Dowsett, A. Ashworth, and L.-A. Martin, "Mechanisms of Disease: angiogenesis and the management of breast cancer," Nature Clinical Practice Oncology, vol. 4, no. 9, pp. 536-550, 2007.

[2] C. Aghajanian, N. J. Finkler, T. Rutherford et al., "OCEANS: a randomized, double-blinded, placebo-controlled phase III trial of chemotherapy with or without bevacizumab (BEV) in patients with platinum-sensitive recurrent epithelial ovarian (EOC), primary peritoneal (PPC), or fallopian tube cancer (FTC)," Journal of Clinical Oncology, vol. 29, supplement 18, 2011, Abstract no. LBA5007.

[3] A. Sandler, R. Gray, M. C. Perry et al., "Paclitaxel-carboplatin alone or with bevacizumab for non-small-cell lung cancer," The New England Journal of Medicine, vol. 355, no. 24, pp. 2542-2550, 2006.

[4] B. Escudier, A. Pluzanska, P. Koralewski et al., "Bevacizumab plus interferon alfa-2a for treatment of metastatic renal cell carcinoma: a randomised, double-blind phase III trial," The Lancet, vol. 370, no. 9605, pp. 2103-2111, 2007.

[5] H. Hurwitz, L. Fehrenbacher, W. Novotny et al., "Bevacizumab plus irinotecan, fluorouracil, and leucovorin for metastatic colorectal cancer," The New England Journal of Medicine, vol. 350, no. 23, pp. 2335-2342, 2004.

[6] H. I. Hurwitz, L. Fehrenbacher, J. D. Hainsworth et al., "Bevacizumab in combination with fluorouracil and leucovorin: an active regimen for first-line metastatic colorectal cancer," Journal of Clinical Oncology, vol. 23, no. 15, pp. 3502-3508, 2005.

[7] G. Kristensen, T. Perren, W. Qian et al., "Result of interim analysis of overall survival in the GCIG ICON7 phase III randomized trial of bevacizumab in women with newly diagnosed ovarian cancer," Journal of Clinical Oncology, vol. 29, no. 18, supplement, 2011, Abstract no. LBA5006.

[8] T. N. Kreisl, L. Kim, K. Moore et al., "Phase II trial of singleagent bevacizumab followed by bevacizumab plus irinotecan at tumor progression in recurrent glioblastoma," Journal of Clinical Oncology, vol. 27, no. 5, pp. 740-745, 2009.

[9] M. Allison, "Avastin's commercial march suffers setback," Nature Biotechnology, vol. 28, no. 9, pp. 879-880, 2010.

[10] A. J. Montero and C. Vogel, "Fighting fire with fire: rekindling the bevacizumab debate," The New England Journal of Medicine, vol. 366, no. 4, pp. 374-375, 2012.

[11] K. Miller, M. Wang, J. Gralow et al., "Paclitaxel plus bevacizumab versus paclitaxel alone for metastatic breast cancer," The New England Journal of Medicine, vol. 357, no. 26, pp. 2666-2676, 2007.

[12] D. W. Miles, A. Chan, L. Y. Dirix et al., "Phase III study of bevacizumab plus docetaxel compared with placebo plus docetaxel for the first-line treatment of human epidermal growth factor receptor 2-negative metastatic breast cancer," Journal of Clinical Oncology, vol. 28, no. 20, pp. 3239-3247, 2010.

[13] N. J. Robert, V. Dieras, J. Glaspy et al., "RIBBON-1: randomized, double-blind, placebo-controlled, phase III trial of chemotherapy with or without bevacizumab (B) for first-line treatment of HER2-negative locally recurrent or metastatic breast cancer (MBC)," Journal of Clinical Oncology, vol. 27, supplement, article 15s, Abstract no. 1005.

[14] D. Moher, A. Liberati, J. Tetzlaff, and D. G. Altman, "Preferred reporting items for systematic reviews and meta-analyses: the PRISMA statement," BMJ, vol. 339, article b2535, 2009.

[15] M. K. Parmar, V. Torri, and L. Stewart, "Extracting summary statistics to perform meta-analyses of the published literature for survival endpoints," Statistics in Medicine, vol. 17, no. 24, pp. 2815-2834, 1998.

[16] J. P. T. Higgins, S. G. Thompson, J. J. Deeks, and D. G. Altman, "Measuring inconsistency in meta-analyses," BMJ, vol. 327, no. 7414, pp. 557-560, 2003.

[17] A. Whitehead, Meta-Analysis of Controlled Clinical Trials, Wiley, Chichester, UK, 2002. 
[18] S. Hapani, D. Chu, and S. Wu, "Risk of gastrointestinal perforation in patients with cancer treated with bevacizumab: a meta-analysis," The Lancet Oncology, vol. 10, no. 6, pp. 559568, 2009.

[19] A. Valachis, N. P. Polyzos, N. A. Patsopoulos, V. Georgoulias, D. Mavroudis, and D. Mauri, "Bevacizumab in metastatic breast cancer: a meta-analysis of randomized controlled trials," Breast Cancer Research and Treatment, vol. 122, no. 1, pp. 1-7, 2010.

[20] J. B. Lee, O. H. Woo, K. H. Park et al., "Bevacizumab for salvage treatment of metastatic breast cancer: a systemic review and meta-analysis of randomized controlled trials," Investigational New Drugs, vol. 29, no. 1, pp. 182-188, 2011.

[21] J. O'Shaughnessy et al., "A meta-analysis of overall survival data from three randomized trials of bevacizumab (BV) and first-line chemotherapy as treatment for patients with metastatic breast cancer (MBC)," Journal of Clinical Oncology, vol. 28, article 15s, 2010, abstract no. 1005.

[22] F. Cuppone, E. Bria, V. Vaccaro et al., "Magnitude of risks and benefits of the addition of bevacizumab to chemotherapy for advanced breast cancer patients: meta-regression analysis of randomized trials," Journal of Experimental and Clinical Cancer Research, vol. 30, no. 1, article 54, 2011.

[23] J. O'Shaughnessy, V. Dieras, J. Glaspy et al., “Comparison of subgroup analyses of PFS from three phase III studies of bevacizumab in combination with chemotherapy in patients with HER2-negative Metastatic Breast Cancer (MBC)," Cancer Research, vol. 69, article 24s, 2009, abstract no. 207.

[24] T. Burzykowski, M. Buyse, M. J. Piccart-Gebhart et al., "Evaluation of tumor response, disease control, progressionfree survival, and time to progression as potential surrogate end points in metastatic breast cancer," Journal of Clinical Oncology, vol. 26, no. 12, pp. 1987-1992, 2008.

[25] K. D. Miller, L. I. Chap, F. A. Holmes et al., "Randomized phase III trial of capecitabine compared with bevacizumab plus capecitabine in patients with previously treated metastatic breast cancer," Journal of Clinical Oncology, vol. 23, no. 4, pp. 792-799, 2005.

[26] A. B. I. Brufsky, V. Smirnov, S. Hurvitz et al., "RIBBON2: a randomized, double-blind, placebo-controlled, phase III trial evaluating the efficacy and safety of bevacizumab In combination with chemotherapy for second-line treatment of HER2-negative metastatic breast cancer," Cancer Research, vol. 69, supplement, article 24, 2009, Abstract no. 42.

[27] H. D. Bear, G. Tang, P. Rastogi et al., "Bevacizumab added to neoadjuvant chemotherapy for breast cancer," The New England Journal of Medicine, vol. 366, no. 4, pp. 310-320, 2012.

[28] G. Von Minckwitz, H. Eidtmann, M. Rezai et al., "Neoadjuvant chemotherapy and bevacizumab for HER2-negative breast cancer," The New England Journal of Medicine, vol. 366, no. 4, pp. 299-309, 2012.

[29] J. Cortes, V. Calvo, N. Ramírez-Merino et al., "Adverse events risk associated with bevacizumab addition to breast cancer chemotherapy: a meta-analysis," Annals of Oncology, vol. 23, no. 5, pp. 1130-1137, 2011.

[30] M. M. An, Z. Zou, H. Shen et al., "Incidence and risk of significantly raised blood pressure in cancer patients treated with bevacizumab: an updated meta-analysis," European Journal of Clinical Pharmacology, vol. 66, no. 8, pp. 813-821, 2010.

[31] S. R. Nalluri, D. Chu, R. Keresztes, X. Zhu, and S. Wu, "Risk of venous thromboembolism with the angiogenesis inhibitor bevacizumab in cancer patients: a meta-analysis," JAMA, vol. 300, no. 19, pp. 2277-2285, 2008.
[32] F. A. B. Schutz, Y. Je, G. R. Azzi, P. L. Nguyen, and T. K. Choueiri, "Bevacizumab increases the risk of arterial ischemia: a large study in cancer patients with a focus on different subgroup outcomes," Annals of Oncology, vol. 22, no. 6, pp. 1404-1412, 2011.

[33] T. K. Choueiri, E. L. Mayer, Y. Je et al., "Congestive heart failure risk in patients with breast cancer treated with bevacizumab," Journal of Clinical Oncology, vol. 29, no. 6, pp. 632-638, 2011.

[34] V. Ranpura, S. Hapani, and S. Wu, "Treatment-related mortality with bevacizumab in cancer patients: a meta-analysis," JAMA, vol. 305, no. 5, pp. 487-494, 2011.

[35] I. E. Smith, J. Y. Pierga, L. Biganzoli et al., "First-line bevacizumab plus taxane-based chemotherapy for locally recurrent or metastatic breast cancer: safety and efficacy in an open-label study in 2251 patients," Annals of Oncology, vol. 22, no. 3, pp. 595-602, 2011.

[36] H. J. Burstein, "Bevacizumab for advanced breast cancer: all tied up with a RIBBON?" Journal of Clinical Oncology, vol. 29, no. 10, pp. 1232-1235, 2011.

[37] R. Dienstmann, F. Ades, K. S. Saini et al., "Benefit-risk assessment of bevacizumab in the treatment of breast cancer," Drug Safety, vol. 35, no. 1, pp. 15-25, 2012. 


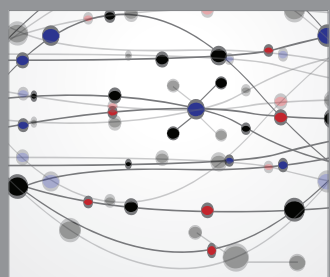

The Scientific World Journal
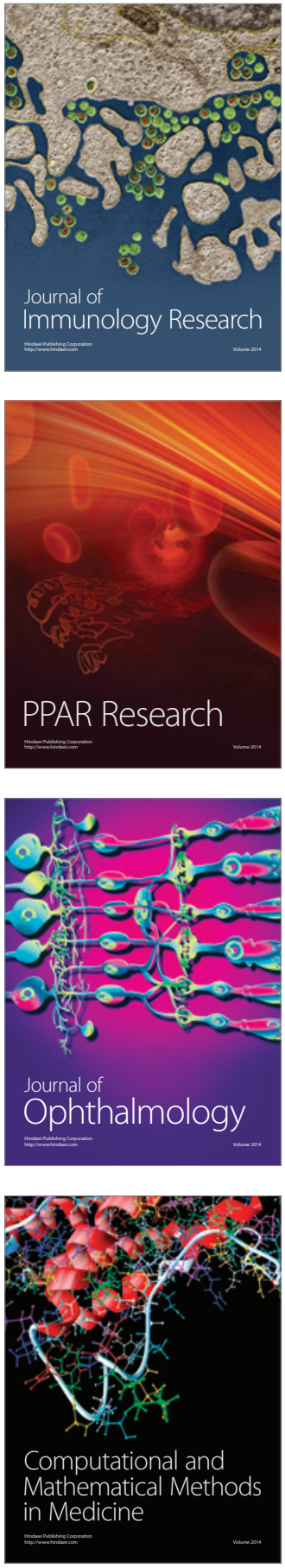

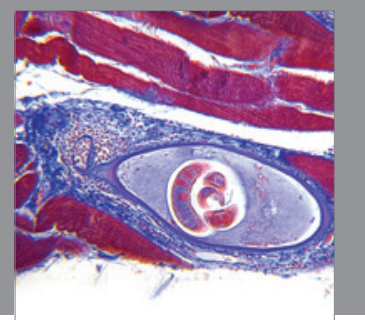

Gastroenterology

Research and Practice
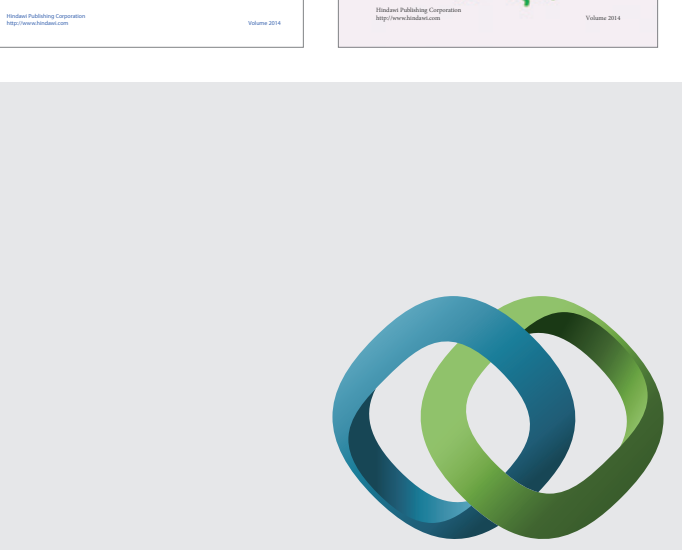

\section{Hindawi}

Submit your manuscripts at

http://www.hindawi.com
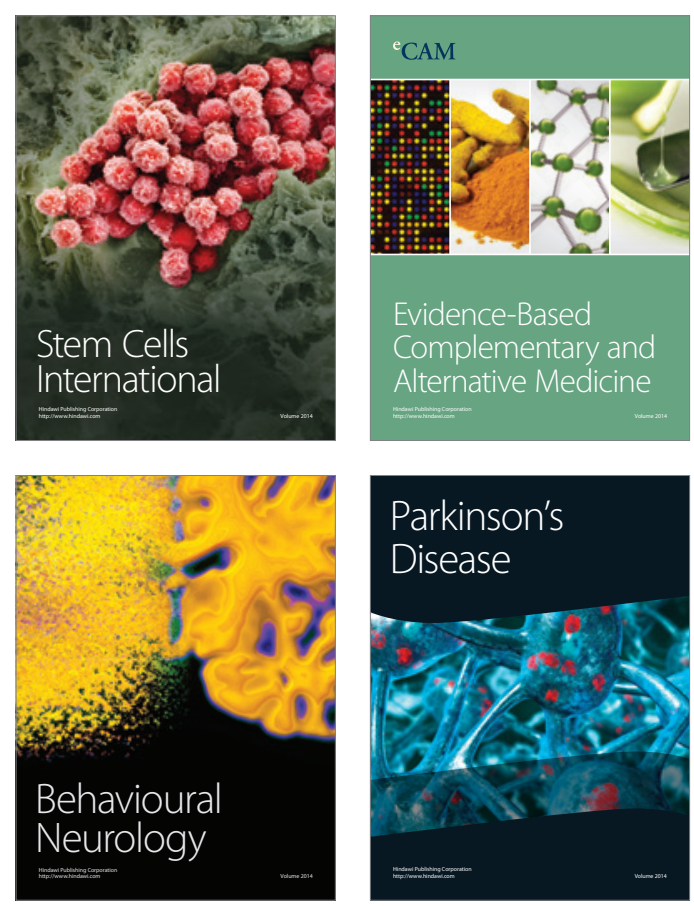

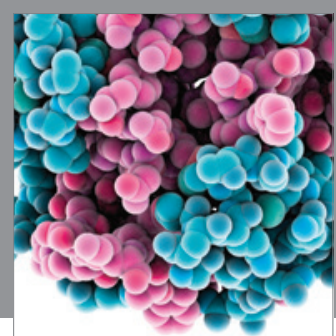

Journal of
Diabetes Research

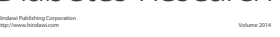

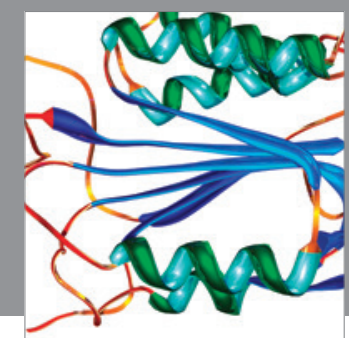

Disease Markers
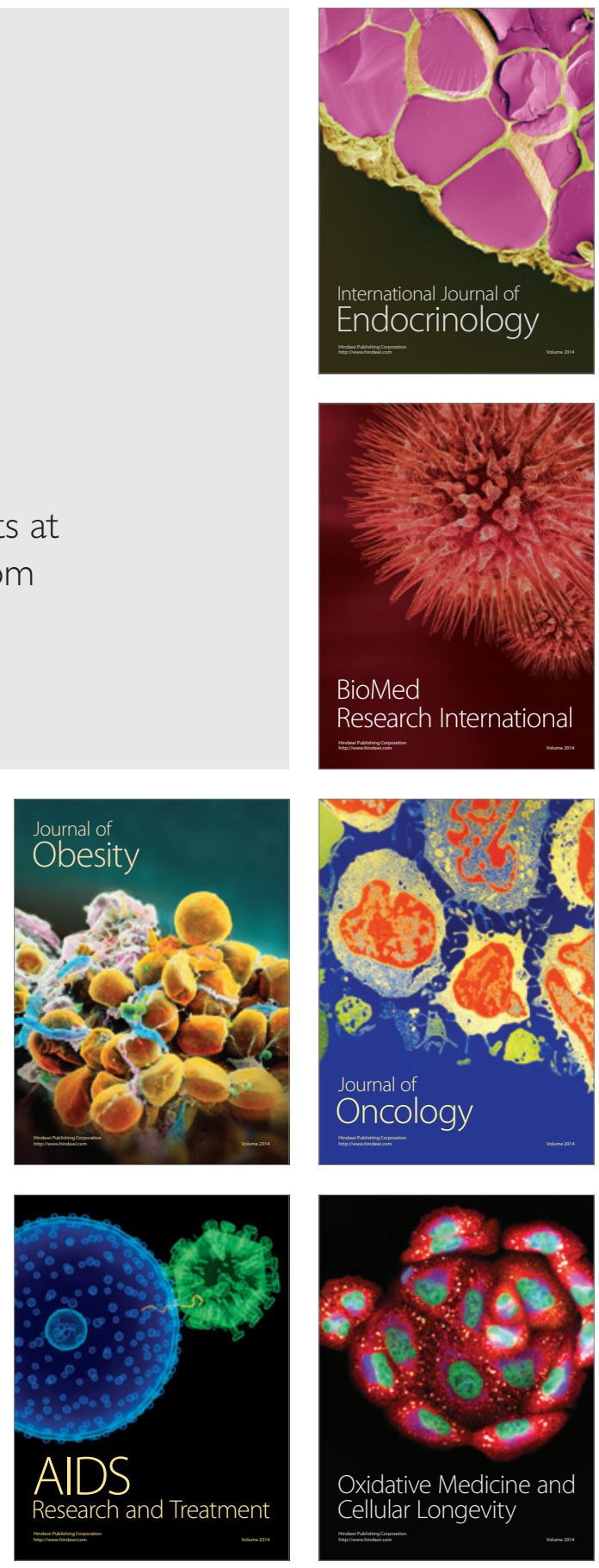